\title{
Isometric Exercises and Mobilization Technique for Patellofemoral Syndrome: Case Report 2019
}

\author{
Azzam Alarab $^{1 *}$, Bayan Abu Shamsiyeh ${ }^{1}$, Alaa Abdo ${ }^{1}$, Ayat Seder ${ }^{1}$, Ratib Abu Shameh ${ }^{1}$, Akram Amro $^{2}$ \\ ${ }^{1}$ Physiotherapy Department, Faculty of Allied Medical Sciences, Palestine Ahliya University, Bethlehem, Palestine \\ ${ }^{2}$ Physiotherapy Department, Faculty of Health Professions, Al-Quds University, Jerusalem, Palestine
}

*Corresponding Author: Azzam Alarab, Physiotherapy Department, Faculty of Allied Medical Sciences, Palestine Ahliya University, Bethlehem, Palestine.

Received: May 14, 2019; Published: June 28, 2019

DOI: 10.31080/ASOR.2019.02.0070

\begin{abstract}
Background: Patellofemoral syndrome (PFS) is a painful sensation in the anterior of the knee joint and around the patella. Objective: To evalute the effectivness of isometric exercises and patellofemoral mobalization techniques of a 32 years old female patient with Patellofemoral syndrome.

Materials and Methods: The study was conducted on physiotherapy clinic at Palestine Ahliya University. The visual analogue scale was used to assess the pain degree, goniometer was used to measure the range of motion of all lower limbs joint, and Oxford scale was used to assess muscle strength of all lower limb muscle. Finally, it was used five functional tests of knee and patellofemoral joints. The physiotherapy management of patellofemoral syndrome has an 8 sessions in a maximal 3 days per week interval which lasts on average 35-45 minute. Each one session included five isometric exercises of knee and hip muscles, such as (Quads Sets, Hamstring set, hip flexor, adductor and abductor). Following this, a patella mobilization was applied of patellofemoral joint.

Results: Eight sessions of physiotherapy management were applied of PFPS symptoms were decreased. All results from pain, muscle strength and range of motion indicated an improvement in the condition of the case after undergoing the treatments.

Conclusion: Individually programmed conservative management based on post isometric exercises techniques and patellar moblization were effective therapy for reducing patellofemoral pain syndrome.
\end{abstract}

Keywords: Patellofemoral Syndrome; İsometric Exercises; Patellar Moblization; Patella

\section{Introduction}

Patellofemoral disorder is a painful condition in the anterior of the knee and around the kneecap. The other name of patellofemoral disorder is "jumper's knee", it regularly influences competitors and the individuals who take part in running, ball, and different games [1]. Your knee is the biggest joint in your body and a standout amongst the most unpredictable changed pivot joint with the best scope of development in flexion and expansion. The knee comprises of two fundamental joints; the femorotibial joint and the patellofemoral joint, which enable the knee to move in three unique planes sagittal, transverse, and frontal [2].

Patellofemoral syndrome is a condition called chondromalacia. Chondromalacia patella is the conditioning and breakdown of the articular ligament on the underside of the kneecap [3]. There are no nerves in articular ligament, so harm to the ligament itself can't legitimately cause pain. Be that as it may, lead to aggravation of the synovium and distress in the basic bone.

Regular reasons for the condition are abuse of the knee joint and injury, for example, hitting the kneecap or falling on it. In spite of the fact that the condition happens all the more regularly in competitors, anybody can have the indications. A few factors that could prompt it. Like, muscular imbalances or weaknesses, especially in the quadriceps muscles at the front of the thigh, tight hamstrings muscle,short ligaments around your knee joint and problems with weight bearing and alignment through your feet. A key factor in PFPS advancement is dynamic valgus of the lower furthest point, which prompts horizontal patellar maltracking. Reasons of dynamic valgus incorporate feeble hip muscles and back foot eversion with pes pronatus valgus. These factors can also be observed in patients with PFOA [4]. 
A significant biomechanical capacity of the patella is that it goes about as a switch, which presents a mechanical preferred position to the quadriceps muscle as in the patella positions for the quad muscle all the more anteriorly, so as to acquire power during augmentation of the leg. Contact of the patella with the femur is started at 20 degrees of flexion and increments with further knee flexion, achieving a most extreme at 90 degrees [5]

A few people have a kneecap that irregular tracks or moves ready toward the finish of the femur. This can cause additional weight on the ligament covering the kneecap or on the femur where it rubs, called the trochlear surface. The patella and trochlea articulation is variable and for some individuals the patella does not fit well [6]. Articular ligament on the underside of the patella enables the patella to coast over the femoral groove, important for productive movement at the knee joint. Overabundance and determined turning powers on the parallel side of the knee can negatively affect the sustenance of the articular ligament and all the more explicitly in the average and focal region of the patella, where degenerative change will happen all the more promptly [7].

The side effect related with patellofemoral disorder is a dull, hurting pain that normally happens on the anterior of the knee. The pain might be in one or the two knees. Extra side effects incorporate; pain when practicing and when twisting the knee, for example, when climbing the stairs, hopping, or hunching down [6]. Distress in the wake of sitting for an all-inclusive time with the knee twisted, for example, while viewing a motion picture at a theater. Splitting or popping sounds in the knee when climbing stairs [8].

As abuse damage, a few hazard components may assume a job in the pathogenesis of PFP. These hazard components are delegated intrinsic and extrinsic hazard factors subsequent to sitting for an all-encompassing time. Extrinsic hazard elements are identified with variables outside the body, for example, the sort and volume of training activity, natural conditions and the surface and hardware utilized. Intrinsic hazard components are ascribed to singular attributes [9].

The diagnosis of patellofemoral syndrome based on your symptoms Physical examination is the foundation of PFP diagnosis. For the most part, your specialist will almost certainly determine patellofemoral pain disorder to have only a physical examination. Much of the time, nonetheless, the individual additionally will arrange a $x$-beam to discount harm to the structure of the knee and to the tissues that interface with it [10].

After examination your physiotherapist can decide the reason for the distress. They may play out some muscle discharges and inform you with respect to explicit stretches to perform. In the event that there is a quality irregularity, you will be given explicit activities went for reinforcing the fitting muscle to lessening any awkwardness. Fortifying project should concentrate especially on the vastus medialis oblique, hip abductors and outer rotators, just as center dependability preparing [11].

Quadriceps strengthening exercise is the most commonly prescribed intervention. Although this approach may represent the therapist perceived "gold" standard, many patients continue to experience pain and dysfunction [12].

Activities and training are two significant parts of a treatment program. Instruction causes the patient to comprehend the condition and how they should manage it for ideal cure. Activities center around extending and reinforcing proper structures, for example, hamstring, quadriceps and gastrocnemius length and quality of the gluteal muscles [13].

Katarzyna Ustarbowska and Bartosz Trybulec, revealed a case report about physiotherapeutic administration of a patient with patellofemoral disorder. As a result they discovered total distress end during day by day exercises was accomplished. Maximal pain power in crushing joint stacking and furthermore clicking and splitting inside the joint happened less frequently and wound up effortless [14]. Isometric activities mean muscle compression without the muscle or joints moving. On the off chance that you push against something that is unfaltering you are encountering isometric withdrawals. This may likewise be called static pressure $[15,16]$.

Moblization technigue is intended to be used as a component of a complete manual therapy program that includes evaluation and treatment of articular, neurovascular, and neuromuscular dysfunctions. The approach encompasses evaluation of the soft tissue system and application of specifically directed manual therapy techniques to facilitate normalization of soft tissue dysfunctions $[17,18]$. This integrated treatment approach has been termed functional mobilization [19].

In the literature, there are few studies on the effect of isometric exercises and mobilization techniques in patients with patellar femoral syndrome. Therefore, the purpose of this study was to compare the effectiveness of isometric exercises and mobilization techniques in patients with patellofemoral syndrome.

\section{Materials and Method}

Case report: The patient is 32 years of age, instructor driving modestly dynamic way of life, She has two daughters and one son. She lives on the fifth floor in an apartment so there are lots 
of stairs. Since 10 months, she was complained from severe pain of the anterior part of the knee joint, the pain increase during long standing, walking and when climbing the stairs. Around 3 months after the first complaint. The patient was administered to 10 sessions of the physiotherapy managementof ultrasound and electrical stimulation technique. $80 \%$ of the symptoms were decreased but after 4 months from treatments, it was returned to appear. İn addition the patient feel cracking or popping sounds in the knee when she is climbing stairs and during pray because; she was do knee flexion about 130 degree. "When you standing for a long time during work, she has feel weakness in your thigh muscles then her knee joint do sudden flexion.

\section{Tests and Measurments}

- Pain assessment: (Visual Analogue Scale), It is a scale developed by Price., et al. it measures the severity of pain in the patient. Scores depend on self-detailed proportions of side effects that are recorded with a individual manually written imprint put at one point along the length of a 10$\mathrm{cm}$ line that speaks to a continuum between the two finishes of the scale-"no pain" on the left end $(0 \mathrm{~cm})$ of the scale and the "most noticeably terrible pain" on the correct end of the scale $(10 \mathrm{~cm})$. The patient is asked to mark a point corresponding to the pain intensity he/she feels on this line. On this case the intensity pain at rest was evaluated as low 4 degree in VAS scale, but was aggravating during climbing stairs, long walking to 8degree in VAS scale [20].

- Range of motion mesurement (goniometer): Goniometer is surely the most prominent instrument to use for estimating joint scope of movement in a clinical setting [21]. This measurement instrument is a helpful, clinical tool that allows for objective measurements in order to accurately track progress in a rehabilitation prograghm. When a patient has a decreased range of motion, a therapist will assess the joint before performing an intervention and will continue to use the tool to make sure that progress is made on this case results the range of motion (ROM) measurements show limited of the Rt knee flexion and hip extention, because the pain is prevention to do normal range of motion.

- The Oxford Scale (muscle manual test): Muscle strength testing is an important component of the physical exam that can reveal information about neurologic deficits. It is used to evaluate weakness and can be effective in differentiating true weakness from imbalance or poor endurance [22]. As indicated by the Oxford scale, muscle quality is reviewed 0 to 5. 0:No motion, grade1: Flicker of motion, grade2: Through full range effectively with gravity, grade3: Through full range effectively against gravity, grade4: Moderated resistance, grade5: Maximum resistance

\section{Functional tests}

- Patellar grind test: The reason for this test is to distinguish the nearness of patellofemoral joint issue (patellofemoral pain, chondromalacia). Patient is situated in supine or long sitting with the included knee expanded. The inspector puts the web space of his hand only better than the patella while applying weight [23]. The patient is told to delicately and progressively contract the quadriceps muscle. A positive sign on this test is pain in the patellofemoral joint. The measure of weight connected must be carefully controlled as more weight can inspire positive reaction even in typical people.

- Patella glide test: This test use to detect normal the mobility of the patella. The patient is lying in supine with knee extention and the examinar place the thumb and index in the fist hand on the base of patella and put other hand on the apex. The examinar do medial and lateral glide of patella. On the off chance that the patella can be moved not exactly a solitary quadrant this might be characteristic of tight parallel delicate tissue structures. In the event that the patella can be moved by more noteworthy than three quadrants, at that point this finding may recommend inordinate patellar motion [24]. Perhaps because of feeble average restrictions. Regardless of this reality, the test is as yet utilized in differential analysis of knee musculoskeletal pathologies.

- Patellar tap test: The patellar tap is a method utilized in an examination of the knee to test for knee effusion. On the off chance that there is swelling in the knee, it ought to be assessed to know whether it is a delicate tissue swelling, a hard swelling or a joint effusion. With the patient lying on their back, the inspector broadens the knee and presses the territory over the kneecap with the palm of one hand. This pushes liquid under the kneecap and lifts it [25]. While keeping the weight on with the direct, the inspector utilizes the fingers of their other hand to press down on the kneecap. The test is sure, if a knee effusion is available, the kneecap will move down and "tap" the bone underneath.

- Thomas test: It was utilized to quantify the adaptability of the hip flexors, which incorporates the iliopsoas muscle group, the rectus femoris, pectineus, gracillus just as the tensor fascia latae and the sartorius. Disabled scope of movement of the hip might be a fundamental reason to different conditions, for example, patellofemoral pain disorder and lower back distress. The patient is approached to lie recumbent. The inspector checks for lordosis which is an indicator of a tight hip flexor. The analyst at that point flexes one hip carrying the knee to the chest and requests that the patient hold the 
knee to help stabilize the pelvis and smooth out the lumbar area [26]. On the off chance that the leg that is being tried the leg on the table does not have a hip flexion withdrawal, it will stay on the testing table. Positive sign show If a contracture is available the leg will raise off of the table. Absence of Full hip augmentation with knee flexion under $45^{\circ}$ demonstrates iliopsoas tightness. On the off chance that full expansion is come to in this position it would shows rectus femoris tightness. On the off chance that any hip outside pivot is watched, it might demonstrate ITB tightness.

- Anterior drawer test: To test the integrity of the front cruciate ligament. The patient's knee is flexed to 90 degrees, and the hip is flexed to 45 degrees. In this position, the foremost cruciate tendon is practically parallel with the tibial plateau. The patient's foot is hung on the table by the analyst's body with the inspector sitting on the patient's forefoot and the foot in neutral position. The analyst's hands are put around the tibia to ensure that the hamstring muscles are loose. The tibia is then drawn forward on the femur. The typical measure of development that ought to be available is roughly $6 \mathrm{~mm}$. In the event that the test is certain (i.e., the tibia pushes ahead more than $6 \mathrm{~mm}$ on the femur), the accompanying structure may have been harmed somewhat; ACL, Posterolateral capsule, Posteromedial capsule, Medial collateral tendon,Iliotibial band, Posterior oblique ligament [27].

- Posterior drawer test: To test the integrity of the back cruciate ligament (PCL). With the patient lying flat and relaxed, the analyst twists the knee to a correct point (90 degrees) [27]. The analyst at that point puts his fingers on the knee joint and endeavors to move the tibia in reverse. As the analyst is setting weight on the highest point of the shin bone, he can feel the opposition that originates from the PCL. In patients with a harmed PCL, the inspector may note extreme interpretation motion of the tibia in reverse, and may not feel the typical opposition of the ligament.

\section{Instrumentations used for treatment}

The duration of the physiotherapy treatment 8 sessions in a maximal 3 days per week interval which lasts on average 35-45 minutes. Each one session included five isometric exercises of knee and hip muscles, such as (Quads Sets, Hamstring set, hip flexor, adductor and abductor). Following this, patella manipulation of patellofemoral joint. After the last session complete practical diagnostics were renewed.

\section{Isometric exercises}

Isometric exercises help reduce pain and improve mobility in patellofemoral joint. This is the primary exercise that a PT will perform to evaluate increase muscle quality and relieve the dimension of pain. The key with isometric exercise is to have no unmistakable motion. Essentially, you are attempting to get a muscle and hold that withdrawal for a couple of moments in a pain unfenced.. Gradually, you should be able to hold the Isometric exercise is one method of muscular exercise. Interestingly, isotonic exercise happens when a contracting muscle shortens against a consistent load, as when lifting a weight [28]. Isometric exercises in detail below.

- Quads sets: The Position of the patient Lying on your back or in long sitting position. Place a rolled towel (or a foam roll) behind the knee. Try to push down the towel with your knee while contracting the quadriceps muscles [29]. Hold it for 3 to 5 seconds in a pain unfenced. Increase the hold to 10 to 15 seconds. Repeat it for 30 times in the treatment session.

- Hamstring set (heel dig): The Position of the patient Lying on your back or in long sitting position with your knee bend $90^{\circ}$. Your good leg should be straight and supported on the floor then try to do hamstring muscle contraction by pressing your heel into the floor [30]. Hold it for 3 to 5 seconds in a pain unfenced. Increase the hold to 10 to 15 seconds. Repeat it for 30 times in the treatment session.

- Hip adductor isometric exercise: The Position of the patient is long sitting with your knees twisted $90^{\circ}$ with a pad set between your knees and your feet level on the floor. Press the pad for 5 seconds and after that release. Hold it for 3 to 5 seconds in a pain unfenced. Increase the hold to 10 to 15 seconds [31]. Repeat it for 30 times in the treatment session.

- Hip abduction isometric exercise: The Position of the patient lying with your knees bent $90^{\circ}$, you can have a $\mathrm{T}$ band or Elastic band around your thighs and try to push your legs outwards, as if you are trying to open your legs outwards. Remember not to have any visible movements and no pain while doing exercises [32]. Hold it for 3 to 5 seconds in a pain unfenced. Increase the hold to 10 to 15 seconds. Repeat it for 30 times in the treatment session.

- Hip flexion isometric exercise: The Postion of the patient Lying on your back or in long sitting with your knee and hip flextion $90^{\circ}$. Try to push your leg upwards against the hand, you are applying resistance in opposite direction with hand on the same leg [33]. Hold it for 3 to 5 seconds in a pain unfenced. 
Increase the hold to 10 to 15 seconds. Repeat it for 30 times in the treatment session.

- Patellofemoral glides (patellar moblization): To reestablish right flexibility of horizontal retinaculum, an inactive average preparation on the patella of second degree. Patellofemoral floats are utilized when confinement of the patellofemoral joint causes pain or diminished in general knee function. Medial/lateral glides are used with the patient in recumbent with the knee marginally flexed. To give a medial glide, the specialist uses two hands to push on the substandard and predominant parts of the medial patella and convey a power to float the patella in a lateral direction [34]. Patella was held in this situation for 15 seconds and after that moved back to the beginning position. This system was rehashed multiple times with a few seconds interval. On the other hand, contact focuses on the lateral patella are utilized to deliver an a medial glide. Superior and inferior glides are utilized for joint play and patellar movement fundamental for augmentation and flexion, separately. Like the medial/lateral glide, joint surfaces in favor of the patella inverse the bearing of assembly are utilized. For instance, inferior surfaces are utilized to prepare the patella a predominant way and the other way around.

\section{Results}

The results of reassessment after the end of the physiotherapy sessions were shown the change of all initially data for the patient, it is positive outcome that shown, the effect of physiotherapy interventions from isometric exercises and patella mobilization to take successful management for patellofemoral syndrome. The pain before the treatment was a 8 degree according to VAS scale especially in the anterior area of the patella, during activity the pain was reach to the peaks such as walking, ascending and descending stair then during long sitting. After physiotherapy management the pain become 0 degree that means pain disappeared on the rest and activity condition. And during isometric exercises the patient was doing that without any uncomfortable felling that's limited of patellofemoral joint motion. The quadriceps muscle strengthening is more relation to relieve patellofemoral pain, because the isometric quadriceps exercise causes improve of biomechanical advantage of patella and decrease pressure on the bone and the cartilage in knee joint, which means no any negative feeling of pain. Table 1 below shown these findings.

The range of motion was limited on the Rt side. In the hip joint extension (7) degrees compared to the other side and the knee flexion is approximately (123) degree.

\begin{tabular}{|l|c|c|}
\hline $\begin{array}{l}\text { Effect pain of the } \\
\text { life style }\end{array}$ & $\begin{array}{c}\text { Pre } \\
\text { intervention }\end{array}$ & $\begin{array}{c}\text { Post } \\
\text { intervention }\end{array}$ \\
\hline Standing & NO & NO \\
\hline Walking & Yes & NO \\
\hline Go up & Yes & NO \\
\hline Go down to stair & NO & NO \\
\hline At night & NO & NO \\
\hline At morning & Yes & NO \\
\hline Jumping & Yes & NO \\
\hline Driver & Yes & NO \\
\hline Squatting & Yes & NO \\
\hline $\begin{array}{l}\text { pain according VAS } \\
\text { scale }\end{array}$ & pre intervention \\
pain & $\begin{array}{c}\text { Post intervention } \\
\text { pain }\end{array}$ \\
\hline
\end{tabular}

Table 1: Pain before and after treatment.

The pain of the knee joint and shortening of the hip flexor muscle is main cause to limit the range from the normal average. The mobilization technique was more effective to increase the flexibility of the patellofemoral joint and the elasticity of the all tissue surrounded the knee joint from ligament, tendon and muscle. After doing the reassessment the range of motion of hip extensor and knee flexor was increased to be normalize that's mean more effective of the physiotherapy treatment. Table 2 below shown these findings.

\begin{tabular}{|l|c|c|c|c|}
\hline \multicolumn{1}{|c|}{ ROM } & $\begin{array}{c}\text { Rt side } \\
\text { pre } \\
\text { inter- } \\
\text { vention }\end{array}$ & $\begin{array}{c}\text { Lt side } \\
\text { Pre } \\
\text { interven- } \\
\text { tion }\end{array}$ & $\begin{array}{c}\text { Differ- } \\
\text { ence in } \\
\text { ROM }\end{array}$ & $\begin{array}{c}\text { Rt side } \\
\text { post } \\
\text { interven- } \\
\text { tion }\end{array}$ \\
\hline Hip flexion & 110 & 110 & 0 & 110 \\
\hline Hip extension & 7 & 14 & 7 & 13 \\
\hline Hip abduction & 45 & 47 & 2 & 45 \\
\hline Hip adduction & 30 & 32 & 2 & 30 \\
\hline $\begin{array}{l}\text { External } \\
\text { rotation }\end{array}$ & 41 & 40 & -1 & 40 \\
\hline Knee flexion & $0-112$ & $0-125$ & 13 & $0-125$ \\
\hline Knee extension & $125-0$ & $125-0$ & 0 & $125-0$ \\
\hline $\begin{array}{l}\text { Ankle planter } \\
\text { flexion }\end{array}$ & 44 & 45 & 1 & 43 \\
\hline Dorsal flexion & 21 & 20 & -1 & 21 \\
\hline Eversion & 10 & 11 & 1 & 10 \\
\hline Inversion & 16 & 17 & 1 & 16 \\
\hline
\end{tabular}

Table 2: ROM before and after treatment. 
The oxford scale shown muscle weakness of the hip adductor flexor and knee extensor muscle as a 3 degree. After performing the four isometric exercise increases the muscle strength to be 4 degrees according to the scale.

The most important point in this report was to focus on the important isometric exercises effect without stimulating the pain or increase the muscle fatigue. But to precisely increase the muscle strength significantly, because its muscle contraction occur without joint movement. Table 3 below shown these findings.

All function tests referred to patient complained from patellofemoral syndrome specially (patella grind test, patella glide test and Thomas test), but the outcome results after the treatment were shown negative result of all previous tests. All results indicated an improvement in the condition of the case after undergoing the treatment. This is confirmed the effective of physiotherapy management to deal the patellofemoral syndromes. Table 4 below shown these findings.

\begin{tabular}{|l|c|c|c|}
\hline \multirow{2}{*}{ M.M.T } & \multicolumn{2}{|c|}{ Pre intervention } & Post intervention \\
\cline { 2 - 4 } & $\begin{array}{c}\text { Oxford Rt } \\
\text { Side }\end{array}$ & $\begin{array}{c}\text { Oxford Lt } \\
\text { Side }\end{array}$ & Oxford Rt Side \\
\hline Hip flexion & 3 & 4 & 4 \\
\hline Hip extension & 4 & 4 & 4 \\
\hline Hip abduction & 4 & 4 & 4 \\
\hline Hip adduction & 3 & 4 & 4 \\
\hline $\begin{array}{l}\text { External } \\
\text { rotation }\end{array}$ & 4 & 4 & 4 \\
\hline Knee flexion & 4 & 4 & 4 \\
\hline $\begin{array}{l}\text { Knee } \\
\text { extension }\end{array}$ & 3 & 4 & 4 \\
\hline $\begin{array}{l}\text { Ankle planter } \\
\text { flexion }\end{array}$ & 4 & 4 & 4 \\
\hline Dorsal flexion & 4 & 4 & 4 \\
\hline Eversion & 4 & 4 & 4 \\
\hline Invertion & 4 & 4 & 4 \\
\hline
\end{tabular}

Table 3: Muscle strength before and after treatment.

\section{Discussion}

In this report was displayed patellofemoral pain disorder and a case of physiotherapeutic administration in a patient with this issue. The goal of study to observed the effect of isometric exercises and patellofemoral mobalization techniques of a 32 years old female patient with PFPS.

This program might be additionally connected in different patients in which confinement of ROM in the knee joint or

\begin{tabular}{|l|c|c|}
\hline Test/Examination & Pre intervention & Post intervention \\
\hline Patellar grind test & Positive & Negative \\
\hline Patella glide test & positive & Negative \\
\hline Thomas test & Positive & Negative \\
\hline Drawer test & Negative & Negative \\
\hline Patella tap test & Negative & Negative \\
\hline
\end{tabular}

Table 4: Test examination before and after treatment.

patellofemoral joint happened based on muscle imbalance. The utilization of treatment that joined different medications relying upon individual elements deciding PFPS, event was considered as a compelling and suggested of treatment. One of the elements included isometric muscle strength exercises.

Rixe JA was reported for, a survey of the administration of patellofemoral pain disorder. The Strength training and stretching exercises continue to be strongly supported by research as effective treatment options for runners with PFPS. Recent studies have confirmed that quadriceps and hip strengthening combined with stretching in a structured physiotherapy program comprise the most effective treatment for reducing knee pain symptoms and improving functionality in patients with PFPS. Additionally, recent research has confirmed that surgical and pharmacologic therapies are not effective for the management of patients with PFPS [35].

This is what the importance of strengthening exercises to management of PFPS. Moreover Chiu JK, was reported for in a review,the impacts of quadriceps reinforcing on distress, capacity, and patellofemoral joint contact in people with patellofemoral syndrome. Weight-preparing activity expanded knee muscle quality and the patellofemoral joint contact, which could diminish mechanical worry in the joint, improving pain and capacity in subjects with PFPS [36].

Lori Bolgla and Terry Malone was reported for, reinforcing of the Quadriceps is a key in the restoration program. pain free activities are significant when treating PFPS. Isometric activities while the knee is completely broadened (patella has no contact with condyles) can be utilized toward the start of the treatment, since it limits weight on the patellofemoral-joint while fortifying the Quadriceps. For example, straight-leg exercise Patient lies on his back, one knee bent at $+/-90^{\circ}$ (pain-free if that knee is affected by PFPS) and foot flat on the ground. The other knee is completely broadened. Patient lifts expanded leg and holds it for 10secs, before unwinding (concentric withdrawal or potentially irregular compression is likewise conceivable, which makes it dynamic). Control that Patient keeps an ordinary lumbar lordosis and does 
not remunerate with his basin. Pad crush work out, Patient sits alright with his trunk supported. The two knees broadened. Place a pillow or towel under one knee that knee might be slightly flexed. Patient attempts to push the cushion/towel in the table by expanding his or her knee [37].

Jayaseelan DJ was reported, the effects of joint mobilization on individuals with patellofemoral pain in a systematic review, joint mobilization appeared to be most effective in improving pain and function when coupled with other interventions, although its discrete effect is unclear due to the reviewed studies' design and reporting [38].

The previous studies agreed with my results, that confirmed the physical therapy was able to reduce pain and improve the function of patellofemoral joint throughout strengthening exercises and patella mobilization. On the other side of the treatment, Heintjes and colleagues were reported which, Pharmacotherapy for patellofemoral pain syndrome, they found, there was only limited evidence for the effectiveness of NSAIDs for short term pain reduction in PFPS. The proof for the impact of glycosaminoglycan polysulphate is clashing and merits further examination. The anabolic steroid nandrolone might be viable, yet is unreasonably disputable for treatment of PFPS [39].

\section{Conclusion}

Individually programmed conservative management based on post isometric exercises and patellofemoral glide were an effective therapy for patellofemoral pain syndrome of a functional nature.

\section{Recommendations}

After the results appear in this case report we recommended for all physiotherapist to focus more during the therapeutic management to use exercises and manual therapy such as isometric exercise and patella moblization, because is very effect to treat patellofemoral syndrome. In addition the isometric exercises help to restore normal alignment of patella in the trochlear surface without effort or deterioration of symptoms. On this report the treatment include one case so it was recommended to use previous application of more cases in different ages and living conditions to monitored the effect of physical therapy management as more accurately.

\section{Conflict of Study}

During research in medical journals was found a difficult to get an effect of isometric exercise as a specific, isometric for quadriceps and hamstring muscles to treat patellofemoral syndrome, because some researchers focus on the effect of muscle strengthening exercises as a general.

\section{Bibliography}

1. Jayne L. "Patellofemoral pain syndrome. Medical news today". (2017).

2. Jawad FA and Michael JG. "Anatomy and Physiology of Knee Stability". Journal of Functional Morphology and Kinesiology (2017).

3. William C and Shiel Jr MD FACP. "Chondromalacia Patella (Patellofemoral Syndrome)". Medicine Net (2017).

4. Wolf P and Ingo R. "Patellofemoral pain in athletes". Open Access Journal of Sports Medicine 8 (2017): 143-154.

5. Sameer D MD., et al. "Management of Patellofemoral Pain Syndrome. Anatomy and Biomechanics". American Family Physician (2007).

6. Janice KL. "Biomechanics and pathomechanic of the patellofemoral joint". International Journal of Sports Physical Therapy11.6 (2016): 820-830.

7. Beeton KS. "Manual Therapy Masterclasses". The Peripheral Joints. Churchill Livingstone (2003): 50-51.

8. Thomee R., et al. "Patellofemoral pain syndrome: a review of current issues”. Sports Medicine 28.4 (1999): 245-262.

9. Powers CM. "Rehabilitation of patellofemoral joint disorders: a critical review". Journal of Orthopaedic and Sports Physical Therapy 28.5 (1998): 345-354.

10. Witvrouw E., et al. "Intrinsic risk factors for the development of anterior knee pain in an athletic population. A two-year prospective study". American Journal of Sports Medicine 28.4 (2000): 480-489.

11. Crossley KM., et al. "Patellofemoral pain". BMJ. 351h3939 (2015).

12. Fulkerson JA. "A Practical guide to understanding and treating patellofemoral pain". American journal of orthopaedics 46.2 (2017): 101-103.

13. Clark DI., et al. "Physiotherapy for anterior knee pain: a randomised controlled trial". Annals of the Rheumatic Diseases 59.9 (2000): 700-704.

14. Katarzyna U and Bartosz T. "Physiotherapeutic management of a patient with patellofemoral pain syndrome - a case report". European Journal of Clinical and Experimental Medicine 16.1 (2018): 68-75. 
15. Nimon G., et al. "Natural history of anterior knee pain: a 14- to 20-year follow-up of nonoperative management". Journal of Pediatric Orthopaedics 18.1 (1998): 118-122.

16. Tepoorten BA. "The piriformis muscle". The Journal of the American Osteopathic Association 69.2 (1969): 150-160.

17. Anitha S and Rajesh S. "Accessory piriformis muscle: An easily identifiable cause of piriformis syndrome on magnetic resonance imaging”. Neurology India 59.5 (2011): 769-771.

18. Johnson GS amd Saliba-Johnson VL. "Functional orthopaedics I: Course outline, San Anselmo, Calif, The Institute of Physical Art, (2005).

19. Johnson GS and Saliba-Johnson VL. "Functional orthopaedics II: Course outline, San Anselmo, Calif, The Institute of Physical Art, (2008).

20. Price DD., et al. "The validation of visual analogue scales as ratio scale measures for chronic and experimental pain". Pain 17 (1983): 45-56.

21. Simon Robert. "An Alternate Method of Range of Motion Measurement". The journal of strength and conditioning research 13.4 (1999).

22. Naqvi A l Sherman. "Muscle Strength Grading- National Center for Biotechnology Information". (2019).

23. Baxter R Pocket. "Guide to Musculoskeletal Assessment, 2nd edition". Copyright, Elsevier Science (USA), (2003).

24. Dixit S., et al. "Management of Patellofemoral Pain Syndrome". American Family Physician 75.2 (2007): 194-202.

25. Clinical examination of the knee, chapter 50, Copyright Elsevier. Ltd, (2013).

26. Mital P. "Thomas test- the orthopedic knowledge network". (2011).

27. Magee DJ. "Orthopedic Physical Assessment: 5th Edition". St. Louis, MO: Saunders Elsevier (2008).

28. William C Shiel Jr., et al. "Medical definition of isometric exercise". Medicine Net, (2018).

29. Adam H, MD. Quad Sets. MyHealth. Alberta.ca Network. (2018).

30. Web: MyHealth.Alberta.ca Network. Hamstring set (2019).

31. Kasper K and Thomas B. "Dynamic Adduction". Research gait. (2017).
32. Bijal S. "Four Isometric Exercises to Reduce Hip and Knee Pain". 24TH (2018).

33. Web Kim Nunley. "Iliopsoas Isometric Exercise”. Ghron (2015).

34. Alsulaimani BH. "Effectiveness of patellar mobilization in patellofemoral pain syndrome". MOJ Orthop Rheumatol 11.1 (2019): 31-33.

35. Rixe JA., et al. "A review of the management of patellofemoral pain syndrome". The Physician and Sportsmedicine 41.3 (2013): 19-28.

36. Chiu JK., et al. "The effects of quadriceps strengthening on pain, function, and patellofemoral joint contact area in persons with patellofemoral pain". American Journal of Physical Medicine and Rehabilitation 91.2 (2012): 98-106.

37. Lori B., et al. "Exercise Prescription and Patellofemoral Pain: Evidence for Rehabilitation". Journal of sport rehabilitation 14.1 (2005): 72-88.

38. Jayaseelan DJ. "The effects of joint mobilization on individuals with patellofemoral pain: a systematic review". Clinical Rehabilitation 32.6 (2018): 722-733.

39. Heintjes E., et al. "Pharmacotherapy for patellofemoral pain syndrome". The Cochrane database of systematic reviews 3 (2004): CD003470.

\section{Volume 2 Issue 7 July 2019}

(C) All rights are reserved by Azzam Alarab., et al. 